\title{
Analysis of the Probability of Appearance of An Operating Error of the Mechatronic Module Using Petri Nets
}

\author{
Maxim Trefilova, Alexey Zhdanov ${ }^{\mathrm{b}}$, Valentin Morozov ${ }^{\mathrm{c}}$ \\ Vladimir st. Gorky on 87, Russian Federation \\ atms@vlsu.ru, bzhdanov@vlsu.ru, crvm@vlsu.ru
}

Keywords: mechatronic module, reliability, Petri net error.

\begin{abstract}
The analysis of the mechatronic module of the reliability and error is an important part of modelling and evaluation of the working capacity of complex systems. In many industrial applications, the failure of the system depends not only on all the error states of the system components, but also on the sequence of appearance of these errors. In this work, we present an alternative methodology for estimating the errors of complex systems. The modelling system is carried out using Petri nets instruments.
\end{abstract}

\section{Introduction}

The increase of the reliability, safety and efficacy of additional methods of diagnosis and prevention of errors become the most important for many technical processes. This task consist in determine the kind of error, as well as all its possible peculiarities, such as the magnitude error, place and time of its appearance. The test procedure is founded on the examination of analytical and heuristic indices and on the complete understanding of the process.

In some systems, the symptoms of possible errors are known in general lines. They can be registered as a causal connection: error - events - symptoms. This qualitative knowledge can be further expressed for the diagnosis in the form of the rules: if "a condition", then "an action is executed." As a condition occurs a symptom, as executable action we have the appearing errors and the taken actions to prevent them. But this approach has not proved its reliability in connection with a non-discrete nature of errors and symptoms [1].

\section{Materials and Methods}

We examine in more detail another approach based on an estimation of reliability. The reliability is an attribute of the system to maintain the ability to implement specified functions. This is a enough complex property, including in turn, attributes such as reliability, durability, maintainability and conservability, depending on the purpose of the system and how it is operated.

During the reliability analysis, particularly at the choice of reliability indexes of the object (system), it is essential to have a solution, which must be taken if the object fails.

The analysis of the mechatronic module (Fig. 1) of the reliability and error is an important part of modelling and evaluation of the working capacity of complex systems. In many industrial applications, the failure of the system depends not only on all the error states of the system components, but also on the sequence of appearance of these errors. Therefore, this situation can not be excluded from the examination.

At present, the analysis of such kind of system error is based either on unreal assumptions or on not applicable for the real production needs. In this work, we present an alternative methodology for estimating the errors of complex systems. The modelling system is carried out using Petri nets instruments. In addition, the principle of counters for calculating the time was applied. 


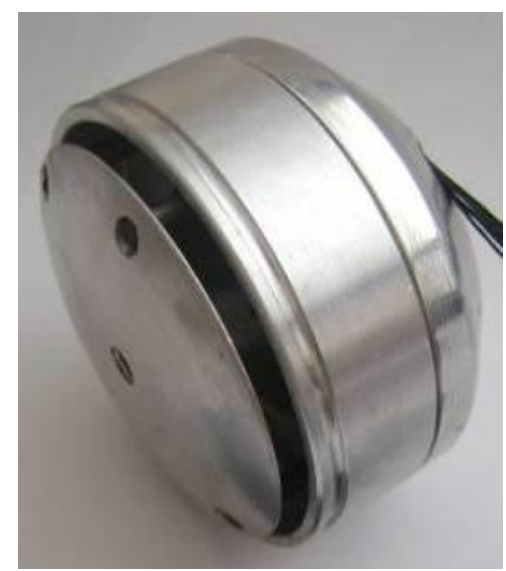

Fig 1 Mechatronic module [2,3]

We examine the model of the dynamics of the mechatronic module for lineal motion [4]. The actuator is placed in a monoblock structure of the drive in a hollow rotor of the electric machine. The dynamics of a mechatronic drive is investigated based on a dual-mass mathematical model.

The system is described by the following system of equations:

$$
\left\{\begin{array}{c}
\dot{\varphi}_{1}=\Omega_{1}, \\
\mu J{ }_{p o t} \dot{\Omega}_{1}=k_{M}\left(U-k_{X} x_{2}-k_{V} v_{2}-k_{a} \dot{v}_{2}\right)-\frac{S_{X}}{\eta_{P B M}} F_{12}-\frac{1}{k_{\omega}} \Omega_{1} \\
\dot{x}_{2}=v_{2}, \\
m_{2} \dot{v}_{2}=F_{12}-k_{p} x_{2}-k_{S} v_{2}-f_{T}\left(v_{2}, F_{12}-k_{p} x_{2}-k_{S} v_{2}\right)
\end{array}\right.
$$

The following disturbance influence on the dynamics of the drive in a big rate:

$\Delta U(t)$ at the input, they are caused by interference of the reverse segments sensors, the electrical losses in the motor windings, the pulsations of his torque, etc.,

$\Delta_{K}(t)$ according to the kinematic errors, they are caused by technological errors of manufacturing, segments deformations during operation process of the drive, etc.,

$F(t)$ in the case of load, they are caused by the load fluctuations and the effect of the environment on the output segment.

Including in the system the terms of disturbances and expressing the coefficients at constants of time and dimensionless coefficients, we obtain a system of equations of the dynamics of the drive:

$$
\left\{\begin{array}{c}
\dot{x}_{1}=y_{1}, \\
\dot{y}_{1}=\frac{v_{0}(t)-y_{1}-\frac{1}{T_{X}} x_{2}-\delta_{V} y_{2}-\frac{F_{12}(\Delta x, t)}{T_{12}}}{T_{\mu}}, \\
\dot{x}_{2}=y_{2}, \\
\dot{y}_{2}=\frac{F_{12}(\Delta x, t)-\delta_{p} x_{2}-T_{s} y_{2}-f_{T}\left(y_{2}, F_{2}\right)-F(t)}{T_{2}^{2}}
\end{array}\right.
$$

where $x_{1}$ is the motion equivalent to the turn of the leading segment at a determined angle $\varphi_{1}, x_{2}$ is the position of the segment, $v_{0}(t)=S_{X} k_{\omega} k_{M}\left[U_{y n p}(t)-u(t)\right]$ is the control velocity, $T_{X}=\frac{1}{S_{X} k_{\omega} k_{M}\left(k_{X}-\frac{k_{a} k_{p}}{m_{2}}\right)}$

is the constant of time of the reverse segment, 
$\delta_{V}=S_{X} k_{\omega} k_{M}\left(k_{V}-\frac{k_{a} k_{S}}{m_{2}}\right)$

$\delta_{p}=\frac{k_{p}}{c}$ is the dimensionless coefficient of the positional load,,$T_{2}=\sqrt{\frac{m_{2}}{c}}$ is the period of the own

oscillations of the load,

$$
T_{12}=\frac{1}{S_{X} k_{\omega} c\left(S_{\eta}-\frac{k_{a} k_{M}}{m_{2}}\right)}
$$

of the segments 1 and 2, $T_{b}=\frac{b}{c}$ is the constant of time of the damping of the transmission, $T_{S}=\frac{k_{S}}{c}$ is the constant of time of the damping of the load, $F_{12}(\Delta x, t)=f_{J}\left(\Delta x-\Delta_{K}(t)\right)+T_{b} \dot{f}_{J}\left(\Delta x-\Delta_{K}(t)\right)$ is the force of elasticity, $F_{2}=f_{J}\left(\Delta x-\Delta_{K}(t)\right)+T_{b} \dot{f}_{J}\left(\Delta x-\Delta_{K}(t)\right)-\delta_{p} x_{2}-F(t)$ are the active forces applied to the output segment of the actuator at $y_{2}=0$. The model of "dry friction" is calculated according to the formula:

$$
f_{T}(v, F)=\left\{\begin{array}{c}
\min \left(F_{A},|F|\right) \cdot \operatorname{sign} F, v=0 \\
{\left[F_{A}-\left(F_{A}-F_{B}\right) \exp \left(-\frac{v_{T}}{|v|}\right)\right] \cdot \operatorname{sign}(v), v \neq 0}
\end{array}\right.
$$

In the model, the output motion is monitored by the sensors of the reverse segments, which translate the deviation of the output coordinates $x_{2}$ to the control voltage.. The sensor is characterized by the coefficient of reinforcement $k_{X}$ and provides a rigid reverse connection on position. Additionally in the system, the reverse connection on velocity $k_{V}$ or the reverse connection on acceleration $k_{a}$ can be presented. The inclusion of these parameters is necessary, as the smoothness of the output output motion depends not only on the magnitude of the output motion, but also on its derivatives.

For compiling the model system, we make use of the Petri nets, which are widely used as a tool for modelling of complex systems. Petri net can be used as a visual mean of connection of components like the flowchart, the block diagram, faults tree and nets. Their use increases the understanding of the relations between different components.

The Petri nets are considered the best alternative of fail-safe analysis, since the net not only graphically represents the causal and effect connections between events, but also it describes the dynamic behaviour of the system. The transition from the faults tree to the representation in the form of Petri nets allows implement an analysis of the reliability and error, as well as an effective way to achieve optimum and minimum paths on the graph. The modelling using Petri nets allows estimate the quality and reliability in case of failures caused by the combination of unplanned errors and their sequences.

\section{Mathematical Model}

The Figure 2 shows a Petri net for a model of the dynamics of the monoblock mechatronic module of linear motion described above.

Structure of the methodology of analysis of a mechatronic module on reliability consists of five main stages. This structure combines the faults tree analysis, the analysis of the state and the consequences of the failures and the dynamic modelling of Petri nets for identify the possible errors, and the sequence of their appearance. To calculate the appearance probability of an error, its necessary to apply the principle of counters of the Petri nets [5]. 


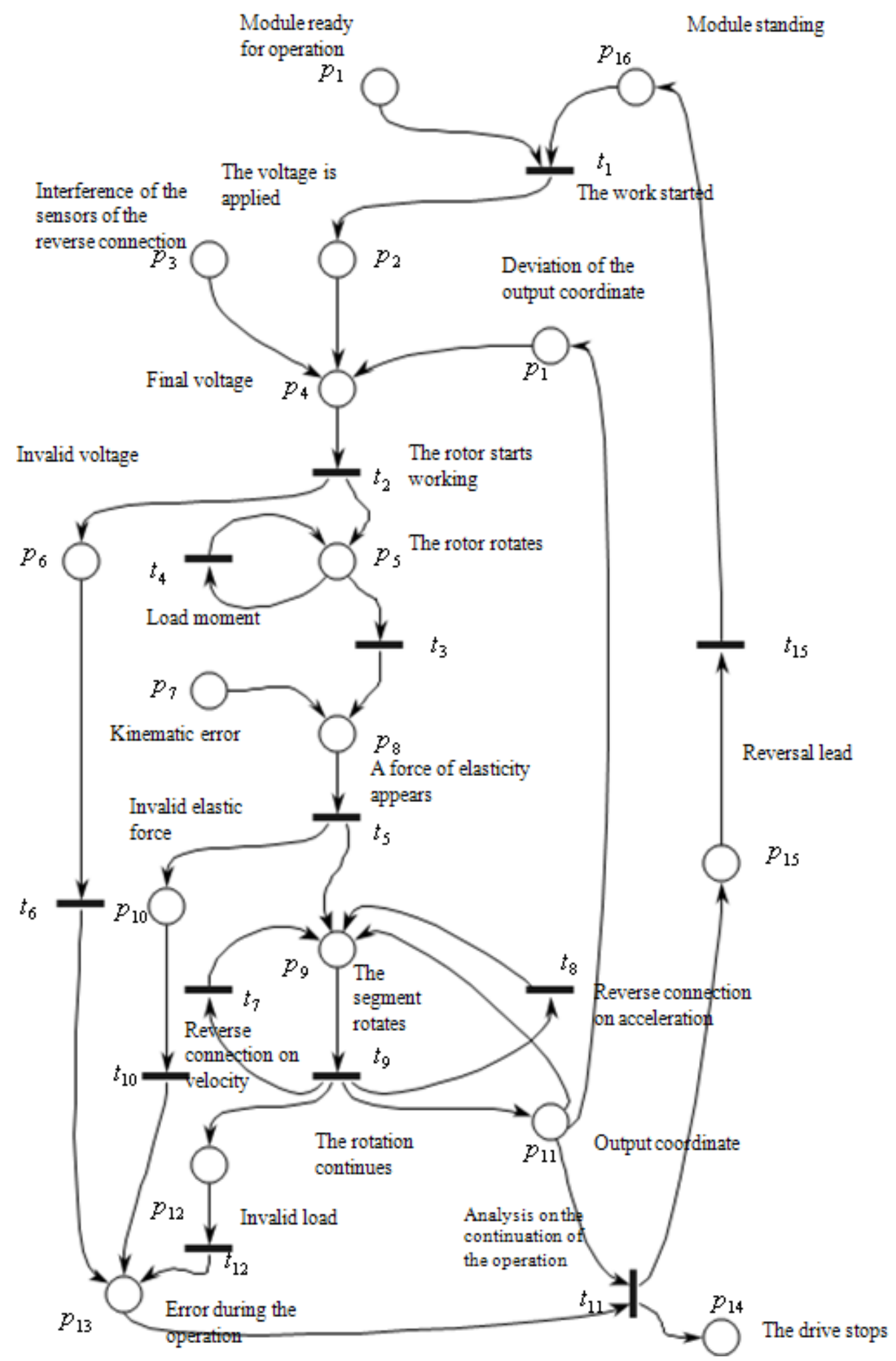

Fig. 2. Petri net

The analysis of errors starts with the error determination of the potential system failures that could cause serious errors in the operation process or in the product. A serious error is the unsatisfactory implementation of actions that appear in the process of the operation or testing. Usually, this analysis can be done using a faults tree, an analysis of the state and the consequences of failure or by other available methods.

The identification of the sequence of the errors begins after built the model as a Petri net of the system subject to the possible failures. If the errors are stochastically and mutually independent with the constant errors and the estimation of repair, then we can use a stochastic Petri net. It is necessary ti 
integrate the errors in a Petri net as follows: the error must be designated as a transition, thee cause of the error must be designated as the input state transition, the consequences of the error as output states.

Based on a Petri net we can build a reachability tree, by the pass with all the available labels on all available transitions starting from the primary label. This procedure is executed as long as all states have been passed. As a result, this representation of the entire system includes the process of its normal operation and all the possible errors. Using the labels, we can determine the sequences of errors by the tracking of the states from the initial label to the label that is in a state of system failure. Thus, it is possible to determine the net routes, which lead to its failure.

Once we defined a sequence of errors, we can calculate the probability of their appearance. As the last transition in the sequence defines a system error, the number of times that this transition has worked for a determines time, and gives us an approximate estimation. In detail, the calculation method is described below.

Principle of determining the probability of error in the system operation based on the use of counters in the Petri net [6].

Let us suppose that it is necessary to calculate the probability that the system will generate an error after 4000 hours, and the lock fails before the operator enters to the danger zone. The values of the operation time of the transitions are listed in Table 1.

Table 1 Time of operation and the mean time of the error transitions

\begin{tabular}{|l|c|c|c|c|c|c|c|c|c|c|c|c|c|}
\hline Operation time & $\lambda_{1}$ & $\lambda_{2}$ & $\lambda_{3}$ & $\lambda_{4}$ & $\lambda_{5}$ & $\lambda_{6}$ & $\lambda_{7}$ & $\lambda_{8}$ & $\lambda_{9}$ & $\lambda_{10}$ & $\lambda_{11}$ & $\lambda_{12}$ & $\lambda_{15}$ \\
\cline { 2 - 13 } & 1 & 0.5 & 0.5 & 0.1 & 0.5 & 0.03 & 0.5 & 0.5 & 1 & 0.02 & 1 & 0.03 & 1 \\
\hline Mean time till the errors & $\phi_{1}$ & $\phi_{2}$ & $\phi_{3}$ & $\phi_{4}$ & $\phi_{5}$ & $\phi_{6}$ & $\phi_{7}$ & $\phi_{8}$ & $\phi_{9}$ & $\phi_{10}$ & $\phi_{11}$ & $\phi_{12}$ & $\phi_{15}$ \\
\cline { 2 - 13 } & 1 & 2 & 2 & 10 & 2 & 2000 & 2 & 1 & 10 & 3000 & 10 & 3333 & 1 \\
\hline
\end{tabular}

In a Petri net are represented at the transition $t_{11}$ two input states $p_{11}$ and $p_{13}$ (Fig. 3). The state $p_{13}$ is not consecutive, therefore, for the calculation of the error on the basis of the previous method, it is necessary to implement a canonical translation, as the Fig. 2 shows. It is introduces a fictitious transition $t_{u}$ and a fictitious state $p_{u}$.

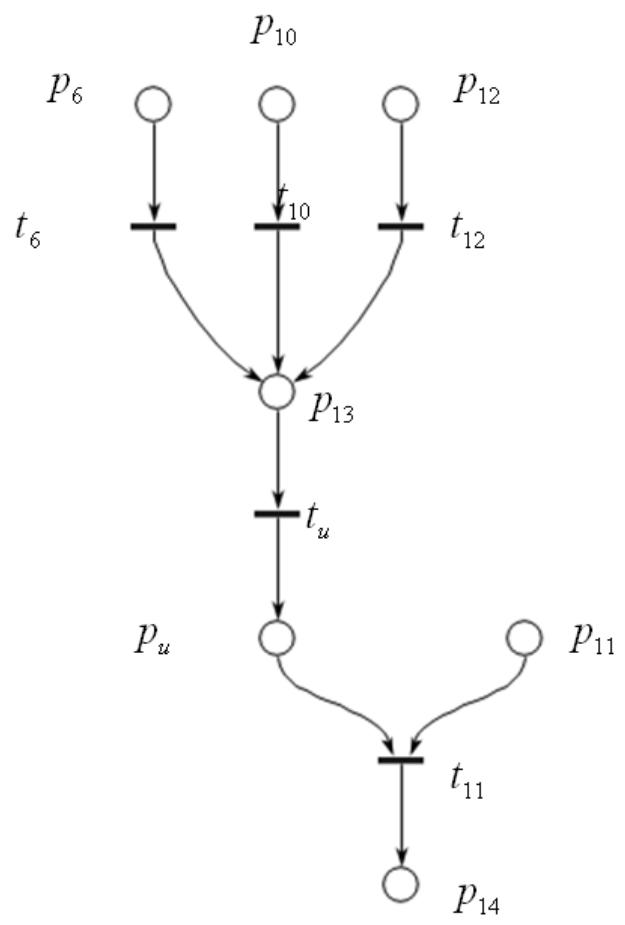

Fig. 2. Canonical translation net in the Fig. 1 
The probability that the transition $t_{u}$ to work off to the point of time $k$, is calculated as $f_{9}(k)=\frac{X_{9}(k)}{k}, X_{9}(k)=\left[\frac{k}{X_{9}\left(k-\phi_{9}-\phi_{6}\right)}\right]$. The probability that the transition will $t_{u}$ work off through the transition ${ }^{t_{6}}$ (invalid voltage) to a point of time $k$, is calculated as

$$
f_{u}^{6}(k)=\frac{X_{6}\left(k-\phi_{6}\right)}{X_{6}\left(k-\phi_{6}\right)+X_{10}\left(k-\phi_{10}\right)+X_{12}\left(k-\phi_{12}\right)} \text {. }
$$

Through 4000 hours, we have gotten a probability of system failure equal to $F^{11}(k)=f_{9}(k) f_{u}^{6}(k)=\left(\left|\frac{\frac{k}{X_{9}\left(k-\phi_{9}-\phi_{6}\right)}}{k}\right|\right)\left(\left|\frac{X_{6}\left(k-\phi_{6}\right)}{X_{6}\left(k-\phi_{6}\right)+X_{10}\left(k-\phi_{10}\right)+X_{12}\left(k-\phi_{12}\right)}\right|\right)=$ $=\left(\left|\frac{1}{\frac{k-\phi_{9}-\phi_{6}}{\phi_{9}}}\right|\right)\left(\left|\frac{\frac{k-\phi_{6}}{\phi_{6}}}{\frac{k-\phi_{6}}{\phi_{6}}+\frac{k-\phi_{10}}{\phi_{10}}+\frac{k-\phi_{12}}{\phi_{12}}}\right|\right)$ $F^{11}(4000)=f_{9}(4000) f_{u}^{6}(4000)=$

$$
\begin{aligned}
& =\left(\left|\frac{1}{\frac{4000-10-2000}{10}}\right|\right)\left(\left|\frac{\frac{4000-2000}{2000}}{\frac{4000-2000}{2000}+\frac{4000-3000}{3000}+\frac{4000-3333}{3333}}\right|\right)= \\
& =\left(\left|\frac{1}{190}\right|\right)\left(\left|\frac{1}{1+\frac{1}{3}+\frac{667}{3333}}\right|\right)=0,003432 .
\end{aligned}
$$

The function $F^{11}(k)$ has a point of rupture in the time of 2010 hours. The rest of the values have an order of 10-2 - 10-3.

The graphic of function until the time of 2100 hours is shown in Fig. 3. after the 2100 hours in the Fig. 4.

To the advantages of the examined method of computing of the probability of error we can attribute: the use of a smaller number of variables; less complex calculations; the possibility to better understand the dynamics of the system; possibility to study huge systems with avoidance of a sharp increase in the conditions space; the time of the error is not limited by the exponential distribution. The built Petri net for examining the system allows present visually the processes of the system and the possible undesirable situations [7]. 


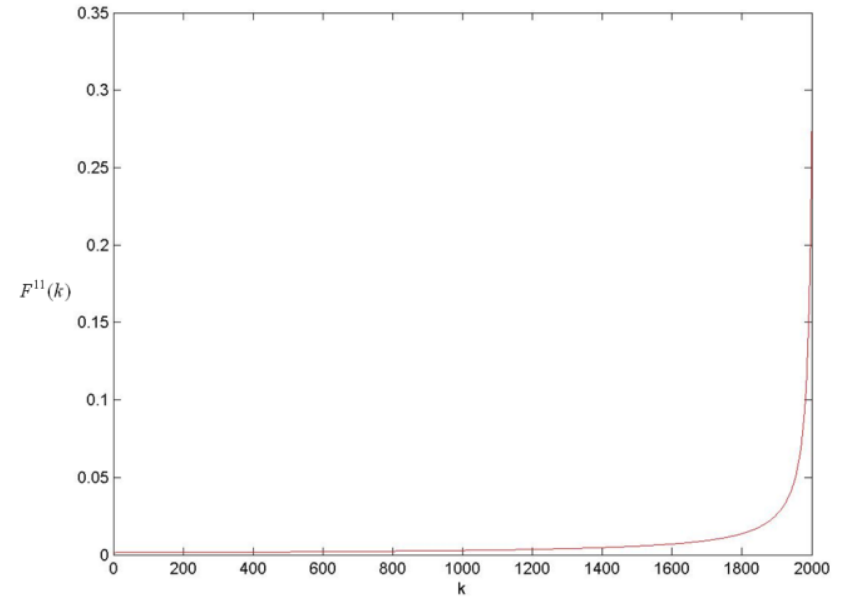

Fig. 3. Graphic of the error probability of the system up to 2100 hours

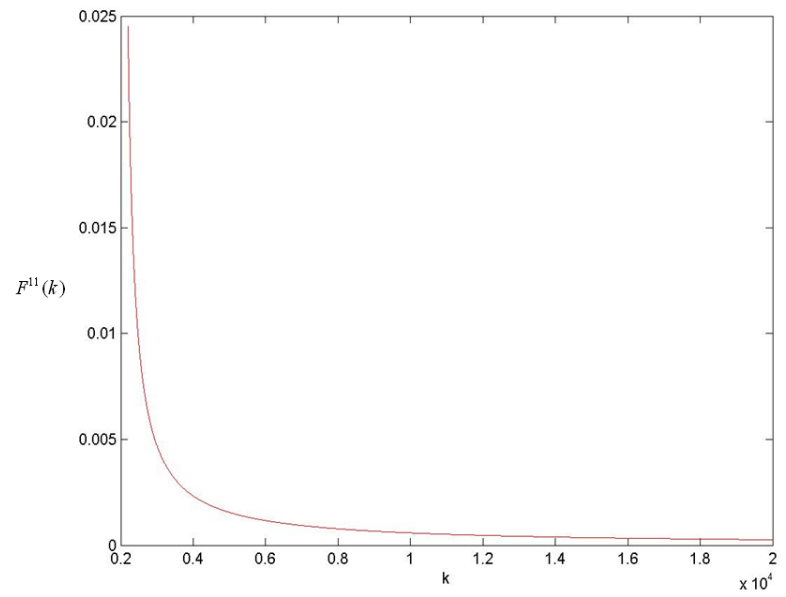

Fig. 4. Graphic of the error probability of the system after 2100 hours

\section{References}

[1] Isermann, R. Model-based fault-detection and diagnosis - status and applications. / R. Isermann // Annual Reviews in Control. - 2005 - №29 - C. 71-85.

[2] Belyaev L.V., Zhdanov A.V., Morozov V.V. Electromechanical drive diaphragm pump of circulatory support (patent) RUS 115664 11.05.2011 (in Russian).

[3] Belyaev L.V., Zhdanov A.V., Morozov V.V. Electromechanical drive diaphragm pump of artificial heart (patent) RUS 104461 06.12.2010 (in Russian).

[4] Morozov, V. V. et al., Smoothness of the dynamic segments of electromechanical drives, Vladimir: Vladimir State University, Vladimir, 1999 (in Russian).

[5] Adamyan, A., He, D. Sequential failure analysis using counters of Petri net models. / A. Adamyan, D. He // Systems, Man and Cybernetics, Part A: Systems and Humans. - 2003 - №33 - C. 1-11.

[6] Morozov V.V., Zhdanov A.V. Electromechanical unit helps artificial heart// Artificial Organs. 1998. -Vol. 22. - № 3. - P. 260-262.

[7] Morozov V.V., Zhdanov A.V., Novikova E.A. Synthesis implantable systems of circulatory support based on mechatronic units // Mechatronica, avtomatizacia, upravlenie, 2007. № 8. p. 1-5 (in Russian) 